\title{
How confocal laser endomicroscopy can help us in diagnosing gastric lymphomas?
}

\author{
Kav $\mathrm{T}^{1}$, Ozen $\mathrm{M}^{1}$, Uner $\mathrm{A}^{2}$, Abdullazade $\mathrm{S}^{2}$, Ozseker B ${ }^{1}$, Bayraktar $\mathrm{Y}^{1}$ \\ Department of Gastroenterology, Hacettepe University Faculty of Medicine, Ankara, Turkey. \\ tkav@hacettepe.edu.tr
}

\begin{abstract}
Primary gastric diffuse large cell lymphoma is one of the most common extranodal lymphomas of the gastrointestinal system. Diagnosing gastrointestinal lymphomas can be difficult, since there is no pathognomonic sign in endoscopy to distinguish it from other malignancies. In some cases biopsy can be non-diagnostic. Therefore, multiple endoscopic examinations and biopsies can be necessary. With using confocal endomicroscopy, histology of the tissue can be seen in vivo and a range of diseases can be identified by using this technique. We are presenting a case, which is diagnosed as primary gastric diffuse large cell lymphoma during the evaluation of erythema nodosum etiology. We want to emphasize the role of confocal laser endomicroscopy for in vivo diagnosis of gastric lymphoma and directing the endoscopist for sampling the diseased mucosa. Confocal endomicroscopy decreases non-diagnostic rates in endoscopic biopsy and can be performed successfully in cases of gastric lymphoma. Pit patterns of gastric lymphoma, ring cell gastric carcinoma and gastric adenocarcinoma are similar. To best of our knowledge, this case is the fifth case of confocal laser endomicroscopy aided in diagnosing gastric lymphomas (Tab. 1, Fig. 2, Ref. 13). Full Text in PDF www.elis.sk.

Key words: confocal laser endomicroscopy, gastric lymphoma, extranodal, endoscopy, pit pattern, erythema nodosum, primary gastric diffuse large cell lymphoma.
\end{abstract}

Extranodal lymphomas are usually detected in gastrointestinal system ( $40 \%$ of cases) and in gastrointestinal system extranodal lymphomas are most commonly seen in stomach ( $75 \%$ of cases) (1). Primary diffuse large B-cell lymphoma (DLBCL) and marginal zone B-cell lymphoma of the mucosa associated lymphoid tissue (MALT) are the most common types of gastric lymphomas (1). Primary gastric lymphomas can be seen as diffusely infiltrative, ulcerative, petechial, hypertrophic or exophytic lesions; however it can be seen as normal mucosa in endoscopy (2-4). About $90 \%$ of lymphomas can be diagnosed by performing endoscopy and biopsy. However, in some cases endoscopy and biopsy can be non-diagnostic or in some cases two or more endoscopic processes may be needed for the diagnosis (3). In submucosal lesions and lymphomas false negative biopsy rates can be $5 \%$ or biopsy results can be nondiagnostic $(5,6)$. To determine the biopsy site during endoscopic examination; new endoscopic techniques have been developed, such as narrow band imaging, high definition endoscopy (7). Confocal endomicroscopy is a new technique that allows distinguishing between benign and malign lesions in gastrointestinal system; therefore it absolutely determines the biopsy site (8).

To best of our knowledge only four cases with gastric lymphoma that were diagnosed by confocal endomicroscopy have been pub-

${ }^{1}$ Department of Gastroenterology, Hacettepe University Faculty of Medicine, Ankara, Turkey, and ${ }^{2}$ Department of Pathology, Hacettepe University Faculty of Medicine, Ankara, Turkey

Address for correspondence: T. Kav, MD, Hacettepe University Faculty of Medicine, Department of Gastroenterology, Sihhiye, Ankara 06100, Turkey. lished. Here we present a case with primary gastric diffuse large B cell lymphoma, which have been diagnosed by confocal endomicroscopy.

\section{Case report}

A 58-year-old woman was admitted to our hospital because of weakness, loss of appetite, stiff redness on her legs for two months and $30 \mathrm{~kg}$ weight loss in 1 year. Physical examination was normal except red nodular lesions on her legs. The lesions were considered as erythema nodosum. Laboratory findings were normal except a high B-2 microglobulin level, for which bone narrow aspiration and biopsy was performed and reported to be normal. To explore stiff redness on her legs, lower extremity Doppler ultrasonography (USG) and echocardiography were done and they were normal. Abdominal USG examination revealed intraabdominal multiple lymphadenopathies (Fig. 1a). Because of diffuse lymphadenopathies and increased gastric antral mural thickening (Fig. 1b) in abdominal computed tomography and dyspepsia; upper gastrointestinal system endoscopy was performed. Endoscopy showed ulceration and nodular appearances at the gastric body extending over the lesser curvature to the antrum (Fig. 2a). Endoscopic biopsies and fine needle aspiration biopsy from abdominal lymph nodes were done. Unfortunately, all biopsies were non-diagnostic and the only histological finding was necrosis.

\section{Confocal laser endomicroscopy process}

Confocal endomicroscopy, which was kindly provided by Cellvizio-GI; Mauna Kea Technologies, Paris, France, for this case, 

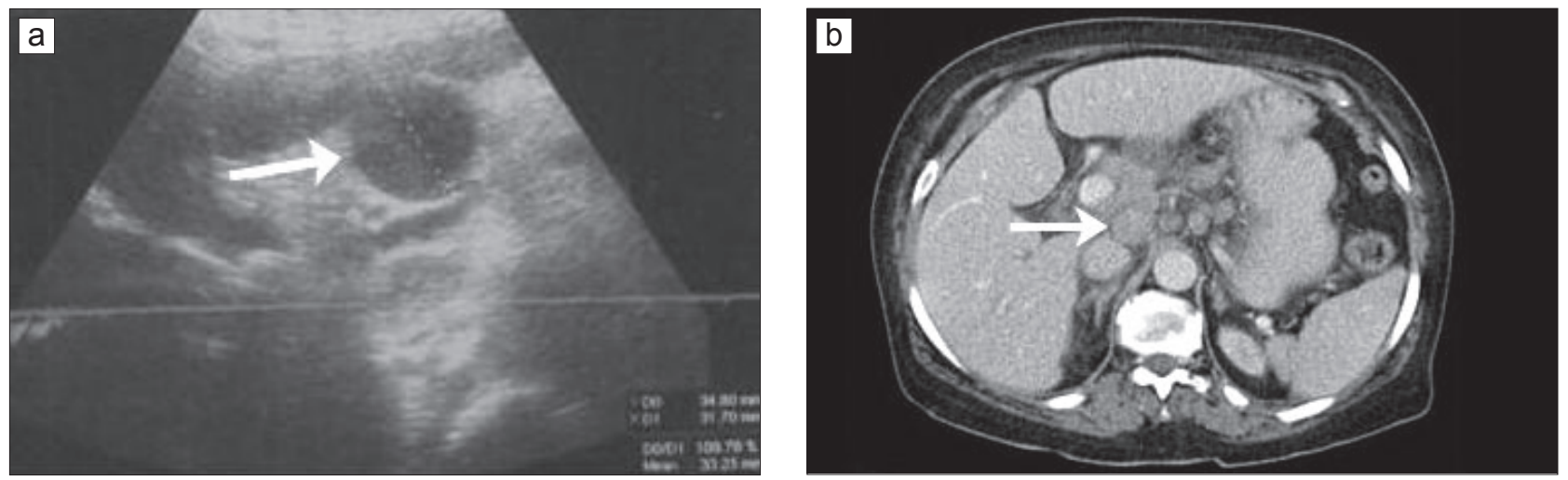

Fig. 1. Arrows show lymphadenopathies detected by (a) abdominal sonogram and (b) computed tomography.
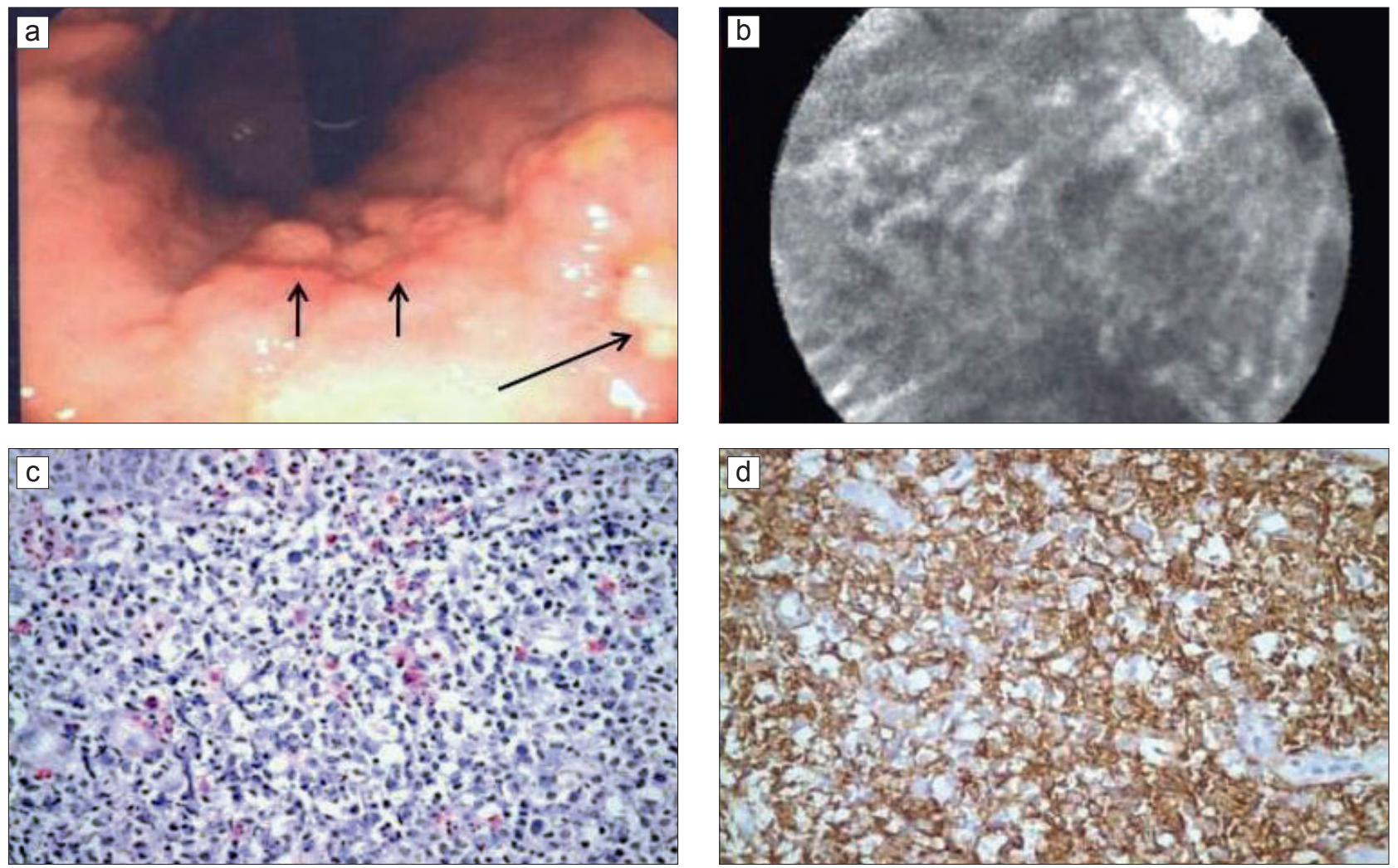

Fig. 2. a) Upper gastrointestinal system endoscopy revealed an ulcer (long arrow) and adjacent mucosal abnormality and nodulation at the gastric body along the lesser curvature, where the random biopsies of this region were negative for malignancy. b) Confocal laser endomicroscopy images were acquired at the corresponding site of the optical endoscopy, where the normal mucosal details were absent and replaced by an irregular structure. c) Histopathologic examination revealed large lymphoid cells with irregular nuclear contours and scant cytoplasm. d) Immunohistochemistry showed these atypical lymphoid cells were strongly positive for CD20.

was performed to show the borders of lesion and determine the nature of the lesion as malignant or benign, and finally to establish the biopsy site. Miniprobe confocal endomicroscopy system was used for this purpose. Lesion was monitored with high definition gastroscope (Olympus GIF-H260; Olympus Optical Co., Tokyo, Japan). Before the miniprobe was passed through, $1 \%$ fluorescein was administered intravenously. Confocal laser microscope having $50 \mu \mathrm{m}$ scanning depth from the epithelial surface was passed through the working channel of the endoscope and placed on the lesion by pressing on it lightly.

Acquired images showed that normal mucosal structure was absent and replaced by an irregular structure (Fig. 2b). We moved the probe along the proximal lesser curvature to see the lesion border and we saw mucosal degeneration on confocal endomicroscopy, but normal on endoscopy. During confocal endoscopy, biopsies were done from irregular sites. 
$680-682$

Microscopic examination of gastric biopsies showed ulceration of the superficial epithelial layer. There was inflammatory cell infiltrate composed of plasma cells, eosinophils and lymphocytes. Randomly distributed large cells with irregular nuclear contours and scant cytoplasm were seen (Fig. 2c). Focal intestinal metaplasia was noted. Immunohistochemically atypical lymphoid cells were strongly positive for CD20 (Fig. 2d), MUM-1 and focally positive for bcl-6. Ki-67 proliferation index was about 70-80\%. The case was diagnosed as Diffuse Large B Cell Lymphoma with these findings.

Six cycles R-CHOP (375 mg/m² Rituximab, 750 mg/m² Cyclophosphamide, $50 \mathrm{mg} / \mathrm{m}^{2}$ Hydroxydaunorubicin (Adriamycin), $1.4 \mathrm{mg} / \mathrm{m}^{2}$ Oncovin (Vincristine), and Prednisolone) chemotherapy regimen was started. After only one cycle chemotherapy skin lesions on legs, considered as erythema nodosum, disappeared.

\section{Discussion}

The principles of confocal imaging were patented by Marvin Minsky in 1961 and have been developed in time (9). Confocal microscopy is a fundamental microscopy technique and is used to get three dimensional and augmented microscopic images. Confocal endomicroscopy is a technique, which mounted confocal microscope to distal part of a conventional endoscope. So, both conventional endoscopy and confocal endomicroscopy images were acquired simultaneously (10-13). Laser light derived from confocal endomicroscope hits the tissue, focused by lenses and reflected light is detected by a photodetector. As a result, only the augmented microscopic images of the focused site are detected, so other images derived from surrounding tissue do not interfere (11-13). Confocal endomicroscopy enables in vivo tissue histology examination, which can be detected better with using contrast agents. First step in the examination of the confocal endomicroscopy is to search for the suspicious mucosal areas, which is usually followed by introduction of confocal endomicroscope through the working channel of the endoscope. Scanning depth of tissue can be adjusted to see deeper structures. If necessary, biopsy can be performed from suspicious areas. Performing biopsy from target area helps getting accurate diagnosis (11).

Confocal endomicroscopic images are classified into 8 types, from $\mathrm{A}$ to $\mathrm{G}$, with using gastric pit pattern by Zhang and coworkers. Type $\mathrm{G}$ is divided to "G1" and "G2". Type A pattern shows normal mucosa; on the other hand, Type G pattern shows malign process; like gastric adenocarcinoma, ring cell gastric carcinomas (12). Our patient's gastric pit pattern was matched with G1 type (Tab. 1).

Confocal endomicroscopy is a useful technique in common gastrointestinal disorders; such as Barrett's esophagus, intestinal metaplasia, amyloidosis and gastric cancer. Currently; colon, pancreas and liver lesions have been scanned with confocal endomicroscopy. Although, by now, confocal endomicroscopy has not replaced the need of biopsy, it is evident that confocal endomicroscopy is helpful to choose the biopsy site $(11,13)$.

Our patient was diagnosed by performing biopsy from target area. Performing biopsy from abnormal areas on confocal endos- copy helped us in determining the correct biopsy region. That area was endoscopically normal. So, accurate diagnosis was done by using confocal endoscopy.

\section{Conclusion}

Confocal endomicroscopy is a useful technique, which decreases non-diagnostic rates in endoscopic biopsy. Confocal endomicroscopy can be performed successfully in cases with gastric lymphoma. Pit patterns of gastric lymphoma, ring cell gastric carcinoma and gastric adenocarcinoma are similar. To distinguish between all of them, biopsy is needed.

\section{References}

1. Psyrri A, Papageorgiou S, Economopoulos T. Primary extranodal lymphomas of stomach: clinical presentation, diagnostic pitfalls and management. Ann Oncol 2008; 19: 1992-1999.

2. Kolve M, Fischbach W, Greiner A, Wilms K. Differences in endoscopic and clinicopathological features of primary and secondary gastric non-Hodgkin's lymphoma. German Gastrointestinal Lymphoma Study Group. Gastrointest Endosc 1999; 49: 307-315.

3. Seifert E, Schulte F, Weismuller J, de Mas CR, Stolte M. Endoscopic and bioptic diagnosis of malignant non-Hodgkin's lymphoma of the stomach. Endoscopy 1993; 25: 497-501.

4. Andriani A, Zullo A, Di Raimondo F et al. Clinical and endoscopic presentation of primary gastric lymphoma: a multicentre study. Aliment Pharmacol Ther 2006; 23: 721-726.

5. Williams DG, Truelove SC, Gear MW, Massarella GR, Fitzgerald NW. Gastroscopy with biopsy and cytological sampling under direct vision. Br Med J 1968; 1: 535-539.

6. Vander Noot MR, Eloubeidi MA, Chen VK et al. Diagnosis of gastrointestinal tract lesions by endoscopic ultrasound-guided fine-needle aspiration biopsy. Cancer 2004; 102: 157-163.

7. Goetz M, Kiesslich R. Advanced imaging of the gastrointestinal tract: research vs. clinical tools? Curr Opin Gastroenterol 2009; 25: 412-421.

8. Kiesslich R, Goetz M, Neurath MF. Confocal laser endomicroscopy for gastrointestinal diseases. Gastrointest Endosc Clin N Am 2008; 18 : 451-466.

9. Minsky M. Microscopy apparatus. United States Patent Office. 1961 Dec 19. US3013467.

10. Kitabatake S, Niwa Y, Miyahara R et al. Confocal endomicroscopy for the diagnosis of gastric cancer in vivo. Endoscopy 2006; 38: 1110-1114.

11. Gheorghe C, Iacob R, Becheanu G, Dumbrav Abreve M. Confocal endomicroscopy for in vivo microscopic analysis of upper gastrointestinal tract premalignant and malignant lesions. J Gastrointestin Liver Dis 2008; 17: 95-100.

12. Zhang JN, Li YQ, Zhao YA et al. Classification of gastric pit patterns by confocal endomicroscopy. Gastrointest Endosc 2008; 67: 843-853.

13. Dunbar K, Canto M. Confocal endomicroscopy. Curr Opin Gastroenterol 2008; 24: 631-637.

Received July 5, 2010. Accepted August 18, 2012. 